\section{Esophageal leakage after surgical repair of sponta- neous rupture: successful closure with endoscopic suture device}

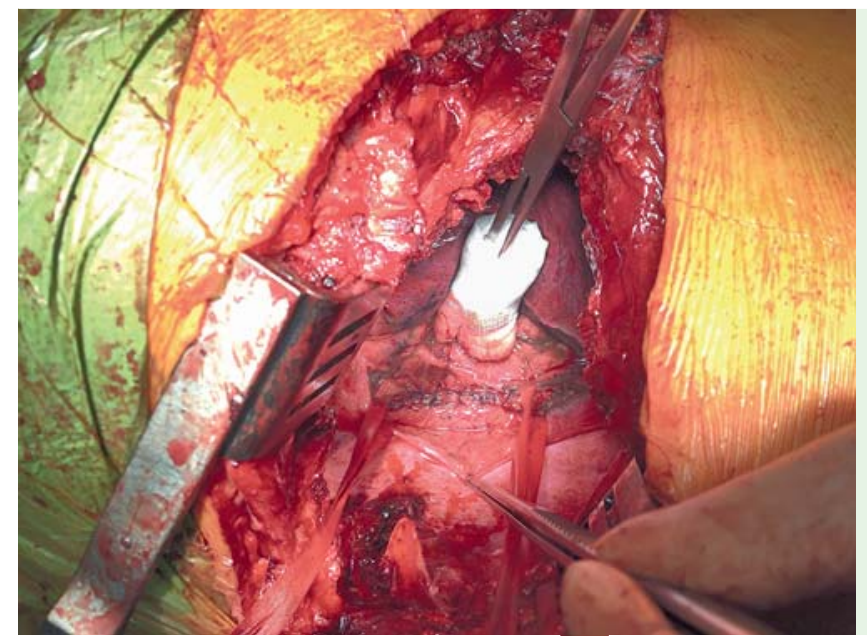

Fig. 1 Surgical repair of spontaneous esophageal rupture in a 60-year-old man: intraoperative appearance.

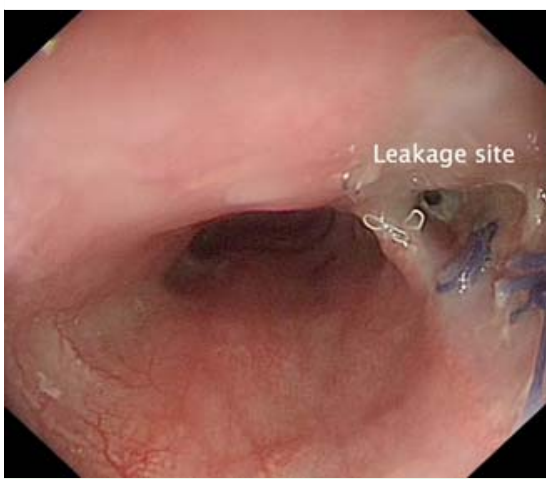

Fig. 2 Endoscopic view of the esophageal leakage site, over the distal end of the repair seen in $\odot$ Fig. 1.

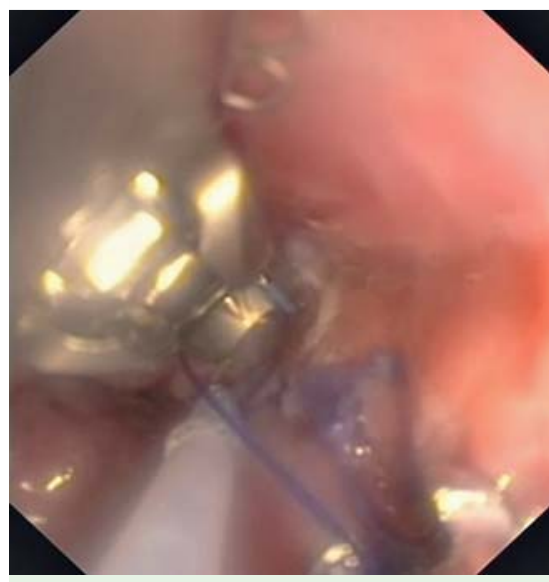

Fig.3 Application of an endoscopic suture device to close the esophageal leakage site.
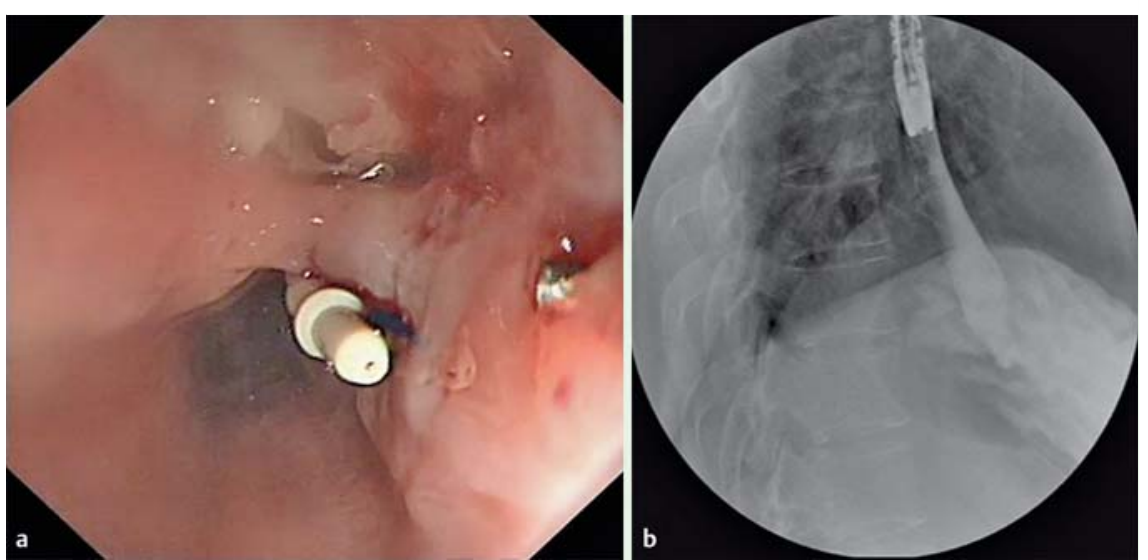

Fig. 4 Follow-up 2 weeks after endoscopic suturing: a endoscopic view; b no contrast leakage shown on fluoroscopy.
We report a case of successful closure of postoperative esophageal leakage using an endoscopic suture device. A 60-yearold man was initially admitted for spontaneous rupture of the esophagus requiring emergency surgery. Intraoperatively a 3-cm perforation was identified on the right side of the lower esophagus. The mucosal defect was first closed with a laparoscopic linear stapler (Endo GIA Ultra; Covidien, Medtronic, Minneapolis, Minnesota, USA) while the muscular defect was approximated with absorbable interrupted sutures $(\bullet$ Fig. 1 ).

He developed fever and leukocytosis again on postoperative day 10 , and computed tomography (CT) of the thorax showed the presence of a right pleural collection and posterior mediastinal gas locules suggestive of leakage. Image guided drainage of the pleural collection was performed.

Esophagoscopy subsequently located the leakage site at the mid-esophagus over the distal end of the previous surgical repair ( $\bullet$ Fig. 2). Two figure-of-eight sutures were applied using an endoscopic suture device (Overstitch; Apollo Endosurgery, Austin, Texas, USA) and good approximation of tissue was achieved ( $\mathrm{Fig.3}$, - Video 1). Later, nasojejunal feeding was initiated.

Repeat endoscopy 2 weeks later showed that stitches from the previous endoscopic intervention had remained in situ and good healing of the leakage site was observed ( $\bullet$ Fig.4a, $\bullet$ Video 1 ). Contrast injection showed no further leakage at the esophagus ( $\bullet$ Fig. 4 b, $\bullet$ Video 1 ). An oral diet was resumed and the patient was discharged home.

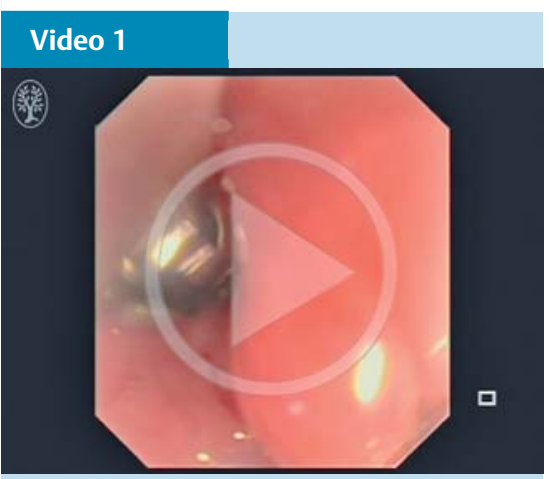

Successful closure, using an endoscopic suture device, of postoperative esophageal leakage following surgical repair of spontaneous rupture of the esophagus. 
Endoscopic closure of postoperative esophageal leakage with endoscopic clips has been reported previously, but its use is limited by the restricted opening distance between the jaws of the clip, the low closure force, and the inability to accomplish deep tissue capture $[1,2]$. Deployment of a covered metallic stent for sealing the leakage site has also been reported, but this method is not guaranteed to completely seal the leak [3]. An endoscopic suture device could achieve full-thickness approximation of tissue, providing a more secure closure of gastrointestinal defects, especially in inflamed tissue such as that around postoperative leakage.

\section{Endoscopy_UCTN_Code_TTT_1AO_2AI}

Competing interests: None. The authors received no funding related to the current research.
Hon Chi Yip, Philip W. Y. Chiu, Shannon M. Chan, Vivien W. Y. Wong, Anthony Y. B. Teoh, Simon K. H. Wong, Enders K. W. Ng ${ }^{1}$

Division of Upper Gastrointestinal and Metabolic Surgery, Department of Surgery, Prince of Wales Hospital, Faculty of Medicine, The Chinese University of Hong Kong, Shatin, Hong Kong

\section{References}

1 ASGE Technology Committee, Banerjee $S$, Barth BA et al. Endoscopic closure devices. Gastrointest Endosc 2012; 76: 244 - 251

2 Shimamoto C, Hirata I, Umegaki E et al. Closure of an esophageal perforation due to fish bone ingestion by endoscopic clip application. Gastrointest Endosc 2000; 51: 736 739

3 Persson S, Rouvelas I, Kumagai K et al. Treatment of esophageal anastomotic leakage with self-expanding metal stents: analysis of risk factors for treatment failure. Endosc Int Open 2016; 4: E420-E426
Bibliography

Dol http://dx.doi.org/

10.1055/s-0042-117275

Endoscopy 2016; 48: E344-E345

(c) Georg Thieme Verlag KG

Stuttgart · New York

ISSN 0013-726X

Corresponding author Philip W. Y. Chiu, MD Department of Surgery 4/F Lui Che Woo Clinical Sciences Building Prince of Wales Hospital Shatin, New Territories Hong Kong Special Administrative Region Fax: +852-26377974

philipchiu@surgery.cuhk.edu.hk 